\title{
Anxiety and Depression in Glaucoma Patients and its Correlations with Vision-related Quality of Life and Objective Visual Function Indices
}

$\mathrm{Na} \mathrm{Wu}$

Eye Institute \& Department of Ophthalmology, Eye \& ENT Hospital, Fudan University https://orcid.org/0000-0001-6485-9656

Xiangmei Kong ( $\square$ kongxm95@163.com )

Eye Institute and Department of Ophthalmology, Eye \& ENT Hospital, Fudan University https://orcid.org/0000-0002-8142-6040

\section{Xinghuai Sun}

Eye Institute and Department of Ophthalmology, Eye \& ENT Hospital, Fudan University

\section{Research}

Keywords: anxiety, depression, vision-related quality of life, objective visual function indices, glaucoma

Posted Date: October 9th, 2020

DOI: https://doi.org/10.21203/rs.3.rs-87542/v1

License: (c) (i) This work is licensed under a Creative Commons Attribution 4.0 International License. Read Full License 


\section{Abstract}

Background: To investigate anxiety and depression in glaucoma patients and its correlations with visionrelated quality of life (VR-QoL) and objective visual function indices.

Methods: In this cross-sectional study, the Hospital Anxiety and Depression Scale (HADS) and the 25-item National Eye Institute Visual Functioning Questionnaire (NEI VFQ-25) questionnaires were administered to 446 Chinese glaucoma patients to assess their psychological disorders, namely anxiety and depression, and VR-QoL, respectively. Socio-demographic and clinical data were collected at the same time. Multivariate linear regression analysis was carried out to investigate the associations between VR-QoL, visual function indices and psychological disorders. Standardized partial regression analysis was used to identify the variable mostly indicative in evaluating psychological burdens.

Results: The prevalence of anxiety and depression in Chinese glaucoma patients was $12.11 \%$ and $25.78 \%$, respectively. Most of the subscales and the composite score of NEI VFQ-25 were negatively associated with each of anxiety and depression after adjusting for socio-demographic and clinical variables. For visual function indices, only the best corrected visual acuity of both eyes was correlated with anxiety after adjustment. Standardized partial regression analysis further showed VR-QoL but not the objective visual function indices mostly relevant to psychological disturbances.

Conclusions: Anxiety and depression were common in Chinese glaucoma patients. Self-reported VR-QoL was beneficial in assessing glaucoma patients' psychological disorders, while objective visual function indices provide little hint on it.

\section{Background}

Glaucoma is the leading cause of irreversible blindness globally. It was estimated that China would account for one quarter in number with glaucoma worldwide [1]. Due to fear of blindness, increased financial burdens [2] and long-term usage of anti-glaucoma medications, patients with glaucoma are more likely to suffer from psychological disturbances compared to the reference subjects [3]. Among them, anxiety and depression are the two most common forms [3]. Glaucoma patients with depressive symptoms have a greater likelihood of developing poorer medication compliance [4].

Glaucoma, as a chronic disease, has long-standing negative effects on patients' vision-related quality of life (VR-QoL) [5]. Decreased QoL is related to increased economic burden on community and healthcare system [5]. QoL has been increasingly considered as an important measure in glaucoma management [6]. Anxiety and depression affect the VR-QoL of glaucoma patients significantly [7].

Although a higher prevalence of anxiety and depression in glaucoma patients, these disturbances have not raised enough clinical research interest. Only a few studies focused on anxiety, depression and their risk factors in glaucoma patients. In this cross-sectional study, we investigated the variables affecting anxiety and depression in Chinese glaucoma patients. Importantly, we found that self-reported VR-QoL 
rather than objective visual function indices, such as visual field (VF) and visual acuity (VA), played the most important role in psychological disturbances.

\section{Methods}

This study was approved by the Ethics Committee of Eye \& ENT Hospital, Fudan University and conformed to the tenets of the Declaration of Helsinki. Written informed consent was obtained from each participant before the enrollment.

Glaucoma patients, including those with primary open angle glaucoma (POAG), primary angle closure glaucoma (PACG) and secondary glaucoma (SG), were recruited consecutively at Eye \& ENT Hospital, Fudan University. Diagnosis of POAG was based on typical glaucomatous disc cupping and compatible VF defects in at least one eye and with the presence of an open angle with a Shaffer grading of $>2$ on gonioscopy. PACG was diagnosed if there was at least 2 quadrants of closed angle where the trabecular meshwork was not visible on gonioscopy. Eyes with secondary causes of glaucoma were diagnosed as SG. Patients with other severe vision-impaired eye diseases, such as age-related macular degeneration, cataract (Lens Opacities Classification System III grade 2 or above), were not eligible to participate in this study. Patients who currently use medications that could have effects on psychiatric assessments were also excluded from the study, e.g., systemic use of beta blockers. In addition, patients with VF defects caused by other ocular diseases other than glaucoma and patients who had incisional eye surgeries or laser treatments within the previous 3 months before the enrollment were not included in the study.

Questionnaires including a checklist of socio-demographic and clinical information, the Chinese versions of the Hospital Anxiety and Depression Scale (HADS) and the National Eye Institute Visual Function Questionnaire 25 (NEI VFQ-25) were distributed to the participants. A neutral interviewer would provide assistance to patients with poor sight or with illiteracy and record their choices.

The HADS was composed of 2 subscales, representing HADS-anxiety (HADS-A) and HADS-depression (HADS-D). It was developed by Zigmond and Snaith to identify and quantify anxiety and depression symptoms in populations with physical illnesses [8]. Each subscale includes 7 questions graded on a 4point Likert scale from 0 to 3 . Thus, the minimum sum score for each subscale is 0 and the maximum is 21 . Higher scores indicate higher degrees of anxiety and depression. As previously described [3,9], the scores of HADS-A and HADS-D above than 10 were defined as anxiety and depression, respectively. The translated Chinese version from the original HADS has been confirmed reliability and validity $[2,10]$.

The NEI VFQ- 25 consists of 25 items related to vision-targeted QoL. These items are grouped into 12 subscales: general health, general vision, ocular pain, near activity, distance activity, social function, mental health, role difficulties, dependency, driving, color vision and peripheral vision. Each subscale has a minimum value of 0 and a maximum value of 100 indicating extreme disability and no disability, respectively. The composite score of the NEI VFQ-25 was the mean score of all the subscales except for general health domain. 
All participants were underwent comprehensive ophthalmologic examinations including the slit-lamp biomicroscopy. Best-corrected visual acuity (BCVA) was measured using the Snellen chart and was transformed to the logarithm of the minimum angle of resolution (logMAR). Intraocular pressure (IOP) was measured by a Goldmann applanation tonometer. Cup-to-disc(C/D) ratio was evaluated according to the fundus photographs by an experienced ophthalmologist. A central $30^{\circ} \mathrm{VF}$ test was examined using the automated static perimetry (Humphrey Visual Field Analyzer $30-2$, Humphrey Instruments, San Leandro, California, USA). VF tests taken within 3 months before the enrollment and with a reliability factor less than $15 \%$ were eligible for the study.

Pearson or Spearman tests were used to assess the correlations between the variables and the psychological disorders. Student's $t$ test, nonparametric test or analysis of variance using Bonferroni post hoc test was carried out for comparisons of subgroups among variables. Multiple linear regression analysis was used to identify the predictive factors for psychological disturbances. Results were summarized as standardized partial regression coefficients. $P$ value $<0.05$ was considered to be statistically significant.

\section{Results}

446 glaucoma patients, including 247 POAG, 168 PACG and 31 SG patients, participated in the study. Among them, POAG was the most common glaucoma type. The socio-demographic and clinical information of the participants were shown in Table 1. The subjects consisted of 232 male and 214 female. The average age of the patients was $57.40 \pm 15.99$ (mean \pm SD) years, ranging from 18 to 91 years. The mean BCVA of the better-seeing eyes was $0.20 \pm 0.29$ and $0.65 \pm 0.76$ for the worse-seeing eyes. The mean MD of the less-damaged eye was $-7.54 \pm 6.07 \mathrm{~dB}$, whereas that of the more-damaged eye was $-13.93 \pm 8.26 \mathrm{~dB}$. 
Table 1

Socio-demographic and clinical characteristics of the participants $(n=$ 446)

\begin{tabular}{|c|c|}
\hline Variables & $\begin{array}{l}\text { Mean } \pm \text { SD } \\
\text { range / number of participants (\%) }\end{array}$ \\
\hline Male & $232(52.02 \%)$ \\
\hline Age, years & $57.40 \pm 15.99(18$ to 91$)$ \\
\hline Living with family & $418(93.72 \%)$ \\
\hline Marital status & $401(89.91 \%)$ \\
\hline Married & $15(3.36 \%)$ \\
\hline Divorced & $30(6.73 \%)$ \\
\hline Single & $31(6.95 \%)$ \\
\hline Education level & $228(51.12 \%)$ \\
\hline Primary school & $187(41.93 \%)$ \\
\hline Middle school & $247(55.38 \%)$ \\
\hline University and above & $168(37.67 \%)$ \\
\hline Type of glaucoma & $31(6.95 \%)$ \\
\hline POAG & $59(13.23 \%)$ \\
\hline PACG & $160(35.87 \%)$ \\
\hline SG & $152(34.08 \%)$ \\
\hline Medication & $75(16.82 \%)$ \\
\hline 0 & $66(14.8 \%)$ \\
\hline 1 type & $193(43.27 \%)$ \\
\hline \multicolumn{2}{|l|}{2 types } \\
\hline \multicolumn{2}{|l|}{3 or more types } \\
\hline \multicolumn{2}{|l|}{ History of laser treatment } \\
\hline \multicolumn{2}{|l|}{ History of surgery treatment } \\
\hline Duration of glaucoma, years & $4.70 \pm 6.36(0.1$ to 37$)$ \\
\hline BCVA of better-seeing eye & $0.20 \pm 0.29(-1.3$ to 2$)$ \\
\hline BCVA of worse-seeing eye & $0.65 \pm 0.76(-0.17$ to 3.2$)$ \\
\hline Lower IOP of both eyes, $\mathrm{mmHg}$ & $16.00 \pm 5.13(6$ to 53.7$)$ \\
\hline
\end{tabular}




\begin{tabular}{|ll|}
\hline Variables & $\begin{array}{l}\text { Mean } \pm \text { SD } \\
\text { range / number of participants (\%) }\end{array}$ \\
\hline Higher IOP of both eyes, $\mathrm{mmHg}$ & $20.40 \pm 9.23$ (7 to 65$)$ \\
Bigger C/D of both eyes & $0.73 \pm 0.20(0.1$ to 1.0$)$ \\
Smaller C/D of both eyes & $0.58 \pm 0.21(0.1$ to 1.0$)$ \\
MD of less-damaged eye, $\mathrm{dB}$ & $-7.54 \pm 6.07(-30.52$ to 0$)$ \\
MD of more-damaged eye, $\mathrm{dB}$ & $-13.93 \pm 8.26(-32.55$ to -0.34$)$ \\
\hline
\end{tabular}

SD: standard deviation; POAG: primary open angle glaucoma; PACG: primary angle closure glaucoma; SG: secondary glaucoma; BCVA: best corrected visual acuity; IOP: intraocular pressure; C/D: cup-to-disc ratio; $\mathrm{MD}$ : mean deviation.

Figure 1 summarized the scores of the 2 questionnaires. The mean subscores of the HADS-A and HADS$D$ in the subjects were $6.11 \pm 3.71$ (ranged from 0 to 18, Fig. 1A) and $7.22 \pm 4.18$ (ranged from 0 to 18 , Fig. 1A), respectively. As previously described, scores above 10 on the HADS-A and HADS-D were defined as anxiety and depression, respectively $[3,9]$. In this study, the prevalence of anxiety and depression in patients with glaucoma was $12.11 \%$ and $25.78 \%$, respectively. The mean composite score of NEI VFQ-25 was $71.52 \pm 15.72$ (range: 14.79 to 97.17 , Fig. 1B). Because of the high missing rate $(308 / 446,69.1 \%$ ), driving had the lowest score among the 12 subscales $(20.97 \pm 34.9)$, while color vision had the highest score (88.62 \pm 21.74$)$. As previously reported [11], the subscale of driving was not included to calculate the composite score of NEI VQF-25.

The univariate associations between socio-demographic, clinical factors and psychological disorders were shown in Table 2. Age and educational level were both statistically significantly correlated with the subscores of HADS-A and HADS-D (all $P<0.05)$. Living status $(P<0.0001)$ and smaller $C / D$ of both eyes $(P=0.007)$ were significantly correlated with anxiety disorders. Statistically significant correlations were observed between marital status $(P=0.038)$, duration of glaucoma $(P=0.004)$, type of glaucoma $(P<$ $0.0001)$, numbers of anti-glaucoma medications $(P<0.0001)$, history of anti-glaucoma surgeries $(P<$ $0.0001)$, higher IOP of both eyes $(P<0.0001)$ and depression disorders. For visual function indices, BCVA of the better-/worse-seeing eyes and MD of the less-/more-damaged eyes were all statistically significantly associated with the subscores of HADS-A and HADS-D (all P<0.05). 
Table 2

Univariate comparisons between socio-demographic, clinical variables and the psychological disturbances

\begin{tabular}{|c|c|c|c|c|}
\hline Variables & HADS-A & & HADS-D & \\
\hline & $\begin{array}{l}\text { Correlation/ } \\
\text { (mean } \pm \text { SD) }\end{array}$ & P value & Correlation/ (mean $\pm S D)$ & $P$ value \\
\hline Age, years & 0.183 & $<0.0001$ & 0.149 & 0.002 \\
\hline Gender & & 0.549 & & 0.354 \\
\hline Male & $6.01 \pm 3.64$ & & $7.04 \pm 4.16$ & \\
\hline Female & $6.22 \pm 3.80$ & & $7.41 \pm 4.21$ & \\
\hline Educational level & & 0.005 & & $<0.0001$ \\
\hline Primary school & $7.71 \pm 4.01$ & & $10.97 \pm 3.56$ & \\
\hline Middle school & $6.34 \pm 3.52$ & & $7.88 \pm 4.00$ & \\
\hline University and above & $5.57 \pm 3.80$ & & $5.80 \pm 3.93$ & \\
\hline Living status & & $<0.0001$ & & 0.847 \\
\hline Living alone & $8.93 \pm 4.17$ & & $7.07 \pm 3.82$ & \\
\hline Living with family & $5.93 \pm 3.61$ & & $7.23 \pm 4.21$ & \\
\hline Marital status & & 0.379 & & 0.038 \\
\hline Married & $6.10 \pm 3.68$ & & $7.33 \pm 4.20$ & \\
\hline Divorced & $7.33 \pm 4.15$ & & $7.87 \pm 4.49$ & \\
\hline Single & $5.73 \pm 3.93$ & & $5.37 \pm 3.48$ & \\
\hline Duration of glaucoma, years & 0.088 & 0.064 & -0.136 & 0.004 \\
\hline Type of glaucoma & & 0.30 & & $<0.0001$ \\
\hline POAG & $6.31 \pm 3.90$ & & $6.25 \pm 4.03$ & \\
\hline PACG & $5.98 \pm 3.54$ & & $8.25 \pm 4.12$ & \\
\hline SG & $5.29 \pm 2.97$ & & $9.35 \pm 3.66$ & \\
\hline Anti-glaucoma medications & & 0.097 & & $<0.0001$ \\
\hline 0 & $5.19 \pm 3.62$ & & $5.63 \pm 3.53$ & \\
\hline 1 & $6.29 \pm 3.81$ & & $6.39 \pm 3.99$ & \\
\hline 2 & $5.99 \pm 3.2$ & & $7.75 \pm 4.23$ & \\
\hline
\end{tabular}




\begin{tabular}{|c|c|c|c|c|}
\hline Variables & HADS-A & & HADS-D & \\
\hline 3 or above & $6.73 \pm 3.66$ & & $9.17 \pm 4.11$ & \\
\hline Laser treatment & & 0.958 & & 0.60 \\
\hline No & $6.11 \pm 3.73$ & & $7.26 \pm 4.22$ & \\
\hline Yes & $6.14 \pm 3.62$ & & $6.97 \pm 3.97$ & \\
\hline Surgery treatment & & 0.749 & & $<0.0001$ \\
\hline No & $6.16 \pm 4.05$ & & $6.37 \pm 3.98$ & \\
\hline Yes & $6.05 \pm 3.22$ & & $8.34 \pm 4.20$ & \\
\hline BCVA of better-seeing eye & 0.189 & $<0.0001$ & 0.209 & $<0.0001$ \\
\hline BCVA of worse-seeing eye & 0.138 & 0.003 & 0.336 & $<0.0001$ \\
\hline Lower IOP of both eyes, $\mathrm{mmHg}$ & 0.001 & 0.988 & -0.011 & 0.824 \\
\hline Higher IOP of both eyes, $\mathrm{mmHg}$ & 0.031 & 0.515 & 0.240 & $<0.0001$ \\
\hline Bigger $C / D$ of both eyes & 0.089 & 0.061 & 0.028 & 0.561 \\
\hline Smaller C/D of both eyes & 0.128 & 0.007 & -0.052 & 0.276 \\
\hline MD of less-damaged eye, $d B$ & -0.178 & $<0.0001$ & -0.157 & 0.001 \\
\hline MD of worsedamaged eye, $\mathrm{dB}$ & -0.1 & 0.035 & -0.223 & $<0.0001$ \\
\hline
\end{tabular}

HADS-A: Hospital Anxiety and Depression Scale-Anxiety; HADS-D: Hospital Anxiety and Depression ScaleDepression; SD: standard deviation; POAG: primary open angle glaucoma; PACG: primary angle closure glaucoma; SG: secondary glaucoma; BCVA: best corrected visual acuity; IOP: intraocular pressure; C/D: cup-to-disc ratio; MD: mean deviation.

Multivariate linear regression analysis was carried out to evaluate the relationships between VR-QoL, objective visual function indices and the psychological disturbances without or with adjustment for socio-demographic and clinical factors. The correlations between the 12 subscales, the composite score of NEI VFQ-25 and the psychological disorders were shown in Fig. 2. After adjustment for variables, the 12 dimensions and the overall score of NEI VFQ-25 were all negatively associated with HADS-A (Fig. 2A). Except dependency $(P=0.187)$ and peripheral vision $(P=0.564)$, the other dimensions and the composite score of NEI VFQ-25 showed negative correlations with HADS-D after adjustment (Fig. 2B). The effects of visual function components on anxiety and depression were shown in Fig. 3. Only BCVA of the better-/worse- seeing eyes was significantly correlated with anxiety after adjustment. There were no statistical relationships between BCVA of the better-/worse- seeing eyes and depression. MD of the 
less-/more- damaged eyes had no statistically significant associations with each of HADS-A and HADS-D after adjustment.

Standardized partial regression analysis was further employed to identify which ones of the visual function indices and VR-QoL played the most important roles in deciding the psychological disturbances. As shown in Table 3, the absolute coefficient value of the composite score of NEI VFQ-25 in HADS-A and HADS-D was 0.65 and 0.39 , respectively (both $P<0.0001$ ), which was the highest and was the only statistically significant deciding factor when compared to objective visual function indices.

Table 3

Results of standardized partial regression analysis to select the variable mostly relevant to HADS-A and HADS-D

\begin{tabular}{|c|c|c|c|c|c|c|}
\hline Variables & HADS-A & & & HADS-D & & \\
\hline & $\begin{array}{l}\text { Regression } \\
\text { coefficient }\end{array}$ & $\begin{array}{l}\text { Standard } \\
\text { error }\end{array}$ & $\mathrm{P}$ & $\begin{array}{l}\text { Regression } \\
\text { coefficient }\end{array}$ & $\begin{array}{l}\text { Standard } \\
\text { error }\end{array}$ & $\mathrm{P}$ \\
\hline NEI VFQ-25 & -0.65 & 0.01 & $\hat{0} .0001$ & -0.39 & 0.01 & $\hat{0} .0001$ \\
\hline $\begin{array}{l}\text { BCVA of the better- } \\
\text { seeing eye }\end{array}$ & -0.07 & 0.59 & 0.14 & 0.02 & 0.68 & 0.62 \\
\hline $\begin{array}{l}\text { BCVA of the worse- } \\
\text { seeing eye }\end{array}$ & -0.10 & 0.25 & 0.07 & 0.03 & 0.29 & 0.59 \\
\hline $\begin{array}{l}\text { MD of the less- } \\
\text { damaged eye }\end{array}$ & 0.04 & 0.04 & 0.55 & -0.03 & 0.04 & 0.67 \\
\hline $\begin{array}{l}\text { MD of the more- } \\
\text { damaged eye }\end{array}$ & 0.02 & 0.03 & 0.74 & 0.02 & 0.03 & 0.72 \\
\hline
\end{tabular}

Standardized partial regression analysis showed when put the composite score of NEI VFQ-25 and the visual function components together, only NEI VFQ- 25 had the most important and statistically significant role in deciding the HADS-A and HADS-D, respectively.

HADS-A: Hospital Anxiety and Depression Scale-Anxiety; HADS-D: Hospital Anxiety and Depression ScaleDepression; NEI VFQ-25: the National Eye Institute Visual Function Questionnaire 25; BCVA: best corrected visual acuity; MD: mean deviation.

\section{Discussion}

The present study examined anxiety and depression in Chinese glaucoma patients and investigated the factors influencing these two psychological disorders. The most important finding of the study was that patients' self-reported VR-QoL other than objective visual function indices, such as MD and BCVA, played the most important deciding role in psychiatric illnesses. 
The prevalence of anxiety and depression in glaucoma patients in our study was $12.11 \%$ and $25.78 \%$, respectively, which was significantly higher than that of the general normal Chinese population $(2.4 \%$ and $1.4 \%$ for anxiety and depression, respectively). It was consistent with previous studies $[9,12,13]$, indicating that glaucoma is a predictor of psychological disturbances. The prevalence of anxiety in glaucoma patients in our study was significantly lower than that in Singapore (64\%) [14] and was similar to that in Turkey (13.5\%) [7] and Japan (13\%) [9]. The prevalence of depression in our study was lower than that in Turkey (57\%) [7] and Singapore (30\%) [14], but was higher than that in Australia (19.09\%) [15], Hungary (12.1\%) [16] and America (10.9\%) [13].

Univariate analysis showed that age had a positive correlation with each of the anxiety and depression in glaucoma patients. The relationships between age and anxiety were controversial. Zhang et al reported that the likelihood of having anxiety along with glaucoma did not change with age [17]. However, other studies revealed that age negatively correlated with anxiety [2,3]. The reasons of the inconsistency could be due to the differences in races, age ranges and numbers of glaucoma patients. In terms of depression, our findings was consistent with previous studies $[3,15]$, which showed that older age was a risk factor for depression. Our results confirmed this finding in a larger range of ages (ranging from 18 to 91 years) in glaucoma patients.

Educational level was another demographic factor that reached statistical significance with both of anxiety and depression. The results implied that increased educational level was along with decreased anxiety and depression symptoms. Living with family related to a decreased anxiety status. In addition, smaller C/D of both eyes was positively associated with anxiety, indicating that worse situation of the better eye signified a more serious anxiety status. A single marital status, long glaucoma course, POAG type, less numbers of glaucoma medications and no history of surgery treatment were related to a low degree of depression. Besides, higher IOP of both eyes was positively correlated with depression.

Multivariate linear regression analysis revealed that the composite score of NEI VFQ-25 and most of the 12 subscales were significantly negatively correlated with each of anxiety and depression disorders after adjusting for socio-demographic and clinical variables. However, for visual function indices, only BCVA of the better- and worse- seeing eyes was significantly related to anxiety after adjustment for other variables and the composite score of VR-QoL. Standardized partial regression analysis further showed that the composite score of NEI VFQ-25 accounted the most important part in each of the HADS-A and HADS-D with statistical significance.

Glaucoma is characterized by progressive optic neuropathy that could lead to visual function damage including VF defects and VA decrease [18]. Ophthalmologists paid close attention to these objective visual function indices in clinical practice. However, our results indicated that patients' self-reported QoL but not visual function components played the most important role in psychological distresses.

Reports about the associations between self-reported measures, objective visual function indices and anxiety in eye diseases were very little. Zhou et al found that worse self-reported visual function assessed by Glaucoma Quality of Life-15 questionnaire (GQL-15) was consistently correlated with decreased 
anxiety and depression in glaucoma patients [2]. In retinitis pigmentosa patients, the degree of anxiety was significantly correlated with the general health and role difficulties of the NEI VFQ-25 dimensions but not with any objective visual functions, such as BCVA of better- and worse- seeing eyes [19]. With regard to depression, our results were consistent with several previous studies. Skalicky et al reported that the total score of GQL-15 was an independent predictor of depression [15]. Jampel et al studied depression and mood indicators in newly diagnosed POAG patients. In compliance with our results, they found that poorer visual function, such as worse VA and more damaged VF, were not correlated with depression symptoms and altered mood. However, patients' perception of their vision in daily-life activities was associated with depression significantly [20]. In a nationally representative glaucoma population, Wang et al observed that several self-reported measures of visual function including NEI VFQ-25 and how much time patients spent worrying about eyesight were significantly associated with depression. Objective measures of glaucoma severity, such as BCVA, MD and C/D, were not predictors of depression [13]. Wilson et al also found that severity of VA and VF were not predictors of depression [21].

To our knowledge, this is an initial Chinese study to find that VR-QoL measured by NEI VFQ-25 was most informative in identifying patients with high risk to suffer from psychological disorders. According to our results, glaucoma patients with reduced VR-QoL are vulnerable to develop anxiety and depression problems. On contrary, patients with good results of objective visual function measures, such as MD and BCVA, do not mean they have a healthy psychological status. Hence, patients with lower VR-QoL but not with lower objective visual function performances should be assessed for potential psychological disorders. Psychological interventions from psychiatrists are needed for glaucoma patients with severe psychological burdens if necessary. For ophthalmologists, except treating patients positively to save their objective visual functions, educating them an overall and detailed knowledge about glaucoma is essential and beneficial. For example, telling patients the slow progression nature of glaucoma and effectiveness in treatments on preventing glaucoma deterioration could alleviate their psychological abnormalities. Our previous study also confirmed that the level on understanding about glaucoma is an independent factor negatively associated with anxiety and depression while positively associated with patients' VR-QoL [22].

This study has several limitations. First, selection bias may be caused because the participants were recruited from a single tertiary institution. Second, even though the HADS questionnaire is common used for study purpose, it could not represent a formal psychological diagnosis of anxiety and depression. In addition, the cross-sectional design of the study made it unable to establish causality. Prospective studies will be needed to further confirm the conclusions.

\section{Conclusions}

Our study showed that self-reported VR-QoL played the most important role in anxiety and depression disorders in glaucoma patients. NEI VFQ-25 is a useful tool to screen glaucoma patients with high risk to suffer from psychological disturbances. This finding help ophthalmologists and psychologists to find patients with psychological disorders early and offer interventions and assistances timely. 


\section{Abbreviations}

BCVA: best corrected visual acuity

C/D: cup-to-disc

GQL-15: Quality of Life-15 questionnaire

HADS: the Chinese versions of the Hospital Anxiety and Depression Scale

HADS-A: Hospital Anxiety and Depression Scale-Anxiety

HADS-D: Hospital Anxiety and Depression Scale-Depression

IOP: intraocular pressure

logMAR: the logarithm of the minimum angle of resolution

MD: mean deviation

NEI VFQ-25: the National Eye Institute Visual Function Questionnaire 25

PACG: primary angle closure glaucoma

POAG: primary open angle glaucoma

SG: secondary glaucoma

VA: visual acuity

VF: visual field

VR-QoL: vision-related quality of life

\section{Declarations}

\section{Ethics approval and consent to participate}

This study was approved by the Ethics Committee of Eye \& ENT Hospital, Fudan University and conformed to the tenets of the Declaration of Helsinki. Written informed consent was obtained from each participant before the enrollment.

\section{Consent for publication}

Not applicable. 
All data generated or analysed during this study are included in this published article and its supplementary information files.

\section{Competing interests}

The authors declare that they have no competing interests.

\section{Funding}

This work was supported by the Surface Project of National Natural Science Foundation of China under Grant 81770922, the project of Shanghai Municipal Commission of Health and Family Planning under Grant 201740204, the clinical science and technology innovation project of Shanghai Shenkang Hospital Development Center under Grant SHDC12017X18, the western medicine guidance project of Shanghai Committee of Science and Technology under Grant 19411961600, Experimental Animal Research Project of Shanghai Science and Technology Commission under Grant 201409006600 and Double Excellence Project of Eye \& ENT Hospital of Fudan University under Grant SYB202003. The funders had no role in study design, data collection and analysis, decision to publish or preparation of the manuscript.

\section{Authors' contributions}

XK and XS conceived and designed the study. NW and XK collected the data, performed the statistical analysis, interpreted the data and drafted the manuscript. All authors read and approved the final manuscript.

\section{Acknowledgements}

We wish to thank all the participants for their time and invaluable devotion.

\section{References}

1. Quigley HA, Broman AT. The number of people with glaucoma worldwide in 2010 and 2020 . Br J Ophthalmol. 2006;90:262-267.

2. Zhou C, Qian S, Wu P, Qiu C. Anxiety and depression in Chinese patients with glaucoma: sociodemographic, clinical, and self-reported correlates. J Psychosom Res. 2013;75:75-82.

3. Mabuchi F, Yoshimura K, Kashiwagi K, Yamagata Z, Kanba S, lijima H, et al. Risk factors for anxiety and depression in patients with glaucoma. Br J Ophthalmol. 2012;96:821-825.

4. Jayawant SS, Bhosle MJ, Anderson RT, Balkrishnan R. Depressive symptomatology, medication persistence, and associated healthcare costs in older adults with glaucoma. J Glaucoma. 2007; 16:513-520.

5. Mills T, Law SK, Walt J, Buchholz P, Hansen J. Quality of life in glaucoma and three other chronic diseases: a systematic literature review. Drugs Aging. 2009;26:933-950. 
6. Onakoya AO, Mbadugha CA, Aribaba OT, Ibidapo OO. Quality of life of primary open angle glaucoma patients in lagos, Nigeria: clinical and sociodemographic correlates. J Glaucoma. 2012;21:287-295.

7. Tastan S, Iyigun E, Bayer A, Acikel C. Anxiety, depression, and quality of life in Turkish patients with glaucoma. Psychol Rep. 2010;106:343-357.

8. Zigmond AS, Snaith RP. The hospital anxiety and depression scale. Acta Psychiatr Scand. 1983;67:361-370.

9. Mabuchi F, Yoshimura K, Kashiwagi K, Shioe K, Yamagata Z, Kanba S, et al. High prevalence of anxiety and depression in patients with primary open-angle glaucoma. J Glaucoma. 2008;17:552557.

10. Leung $\mathrm{CM}, \mathrm{Ho} \mathrm{S}$, Kan CS, Hung $\mathrm{CH}$, Chen $\mathrm{CN}$. Evaluation of the Chinese version of the Hospital Anxiety and Depression Scale. A cross-cultural perspective. Int J Psychosom. 1993;40:29-34.

11. Suzukamo Y, Oshika T, Yuzawa M, Tokuda Y, Tomidokoro A, Oki K, et al. Psychometric properties of the 25-item National Eye Institute Visual Function Questionnaire (NEI VFQ-25), Japanese version. Health Qual Life Outcomes. 2005;3:65.

12. Chan EW, Chiang PP, Liao J, Rees G, Wong TY, Lam JS, et al. Glaucoma and associated visual acuity and field loss significantly affect glaucoma-specific psychosocial functioning. Ophthalmology. 2015;122:494-501.

13. Wang SY, Singh K, Lin SC. Prevalence and predictors of depression among participants with glaucoma in a nationally representative population sample. Am J Ophthalmol. 2012;154:436-444 e2.

14. Lim NC, Fan CH, Yong MK, Wong EP, Yip LW. Assessment of Depression, Anxiety, and Quality of Life in Singaporean Patients With Glaucoma. J Glaucoma. 2016;25:605-612.

15. Skalicky S, Goldberg I. Depression and quality of life in patients with glaucoma: a cross-sectional analysis using the Geriatric Depression Scale-15, assessment of function related to vision, and the Glaucoma Quality of Life-15. J Glaucoma. 2008;17:546-551.

16. Hollo G, Kothy P, Geczy A, Vargha P. Personality traits, depression, and objectively measured adherence to once-daily prostaglandin analog medication in glaucoma. J Glaucoma. 2009;18:288292.

17. Zhang X, Olson DJ, Le P, Lin FC, Fleischman D, Davis RM. The Association Between Glaucoma, Anxiety, and Depression in a Large Population. Am J Ophthalmol. 2017;183:37-41.

18. Yuki K, Awano-Tanabe S, Ono T, Shiba D, Murata H, Asaoka R, et al. Risk Factors for Motor Vehicle Collisions in Patients with Primary Open-Angle Glaucoma: A Multicenter Prospective Cohort Study. PloS one. 2016;11:e0166943.

19. Sainohira M, Yamashita T, Terasaki H, Sonoda S, Miyata K, Murakami Y, et al. Quantitative analyses of factors related to anxiety and depression in patients with retinitis pigmentosa. PloS one. 2018;13:e0195983.

20. Jampel HD, Frick KD, Janz NK, Wren PA, Musch DC, Rimal R, et al. Depression and mood indicators in newly diagnosed glaucoma patients. Am J Ophthalmol. 2007;144:238-244. 
21. Wilson MR, Coleman AL, Yu F, Fong Sasaki I, Bing EG, Kim MH. Depression in patients with glaucoma as measured by self-report surveys. Ophthalmology. 2002;109:1018-1022.

22. Kong XM, Zhu WQ, Hong JX, Sun XH. Is glaucoma comprehension associated with psychological disturbance and vision-related quality of life for patients with glaucoma? A cross-sectional study. BMJ open. 2014;4:e004632.

\section{Figures}
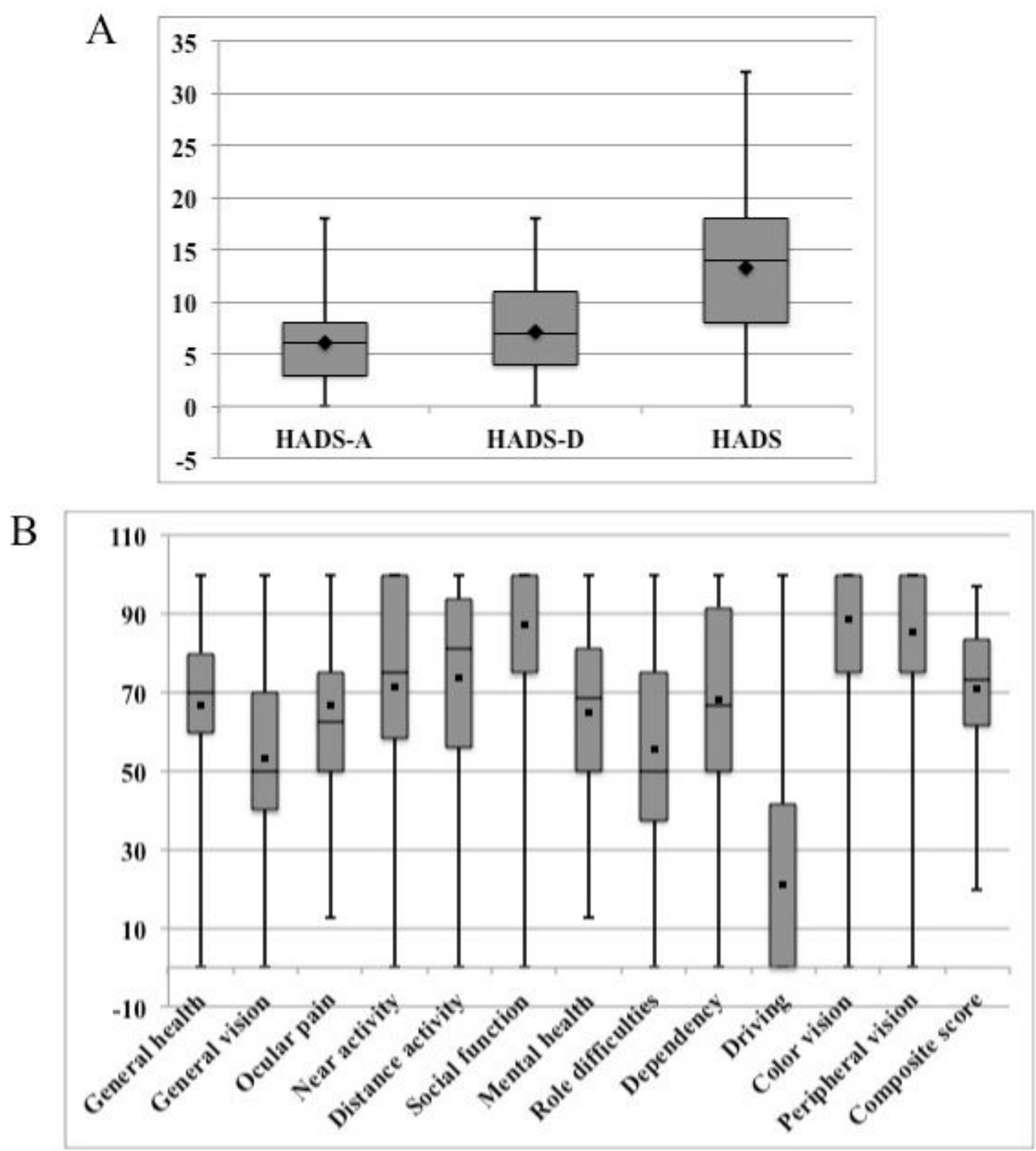

\section{Figure 1}

The average scores of the HADS (A) and the NEI VFQ-25 (B). Black squares and thin error bars represent means and maximum/minimum values, respectively. HADS-A: Hospital Anxiety and Depression Scale- 
Anxiety; HADS-D: Hospital Anxiety and Depression Scale-Depression; HADS: Hospital Anxiety and Depression Scale; NEI VFQ-25: National Eye Institute Visual Function Questionnaire 25.
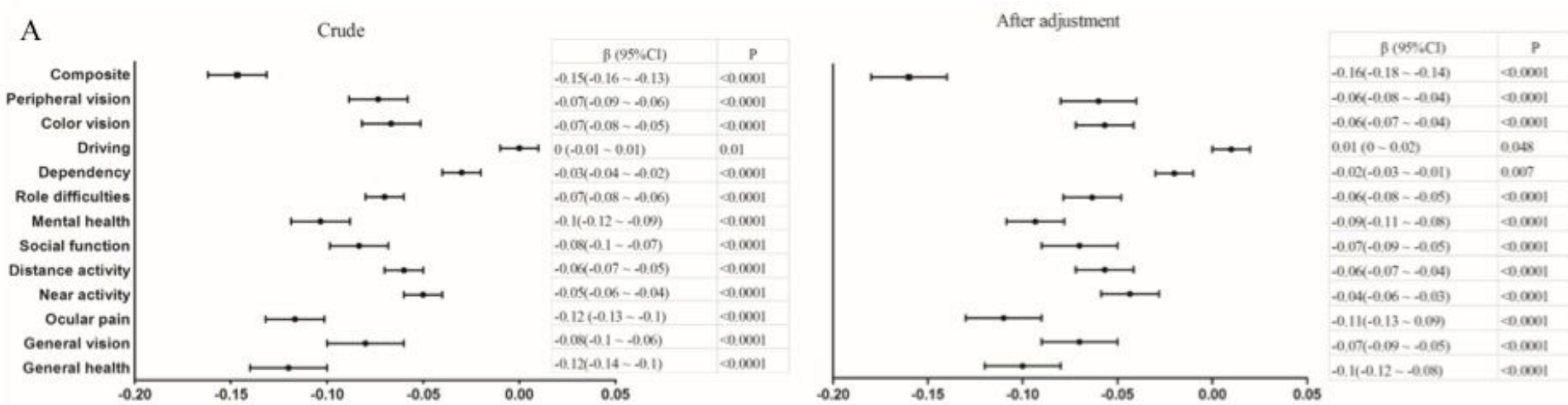

B

Crude
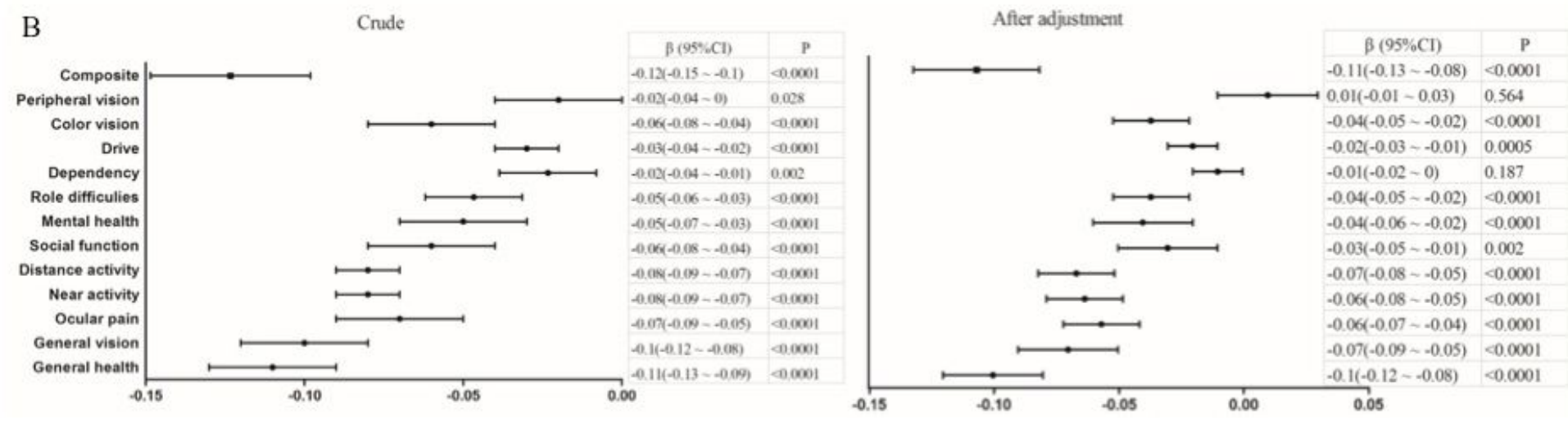

Figure 2

The effects of the subscales and the composite score of NEI VFQ-25 as an independent factor on HADS-A $(A)$ and HADS-D (B) by multivariate linear regression analysis without (Crude) or with adjustment (After adjustment) for socio-demographic and clinical variables. NEI VFQ-25: National Eye Institute Visual Function Questionnaire 25; HADS-A: Hospital Anxiety and Depression Scale-Anxiety; HADS-D: Hospital Anxiety and Depression Scale-Depression. 
A

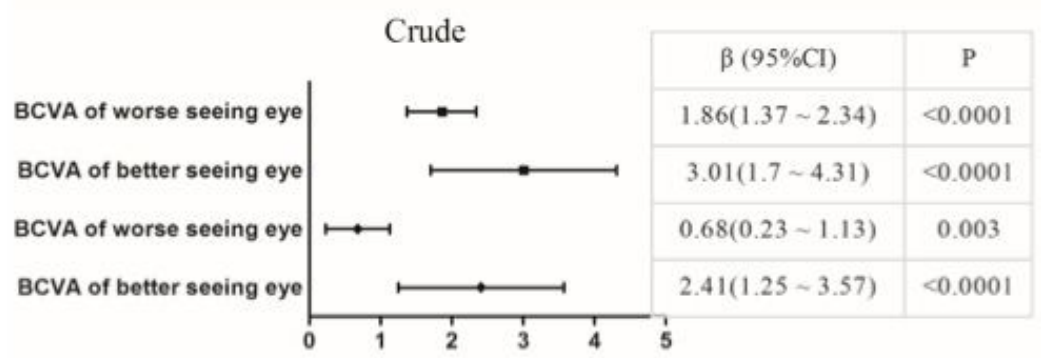

B

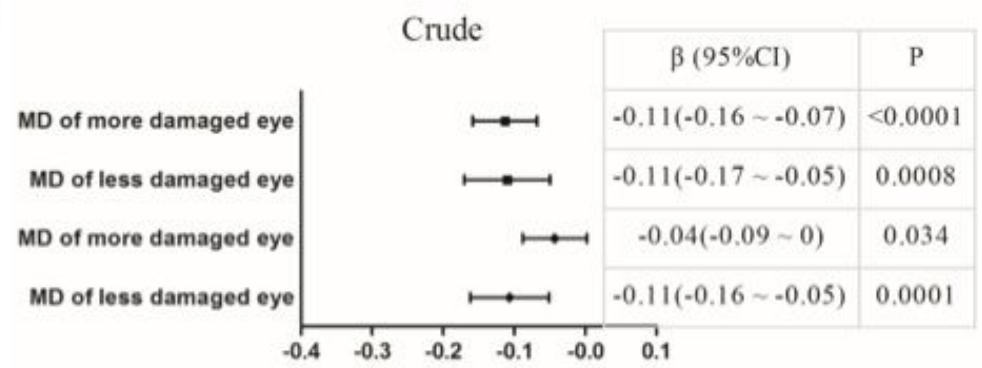

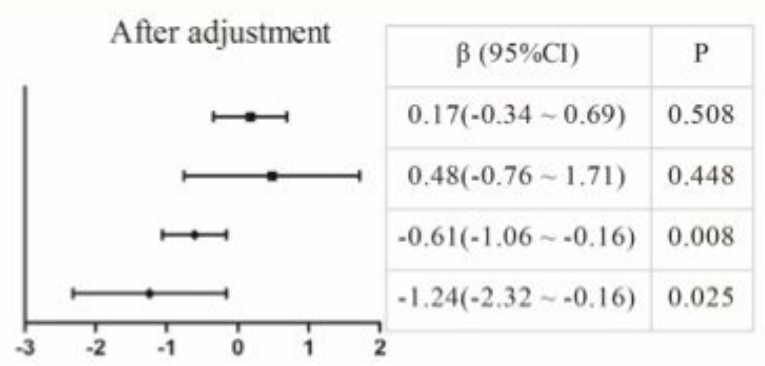

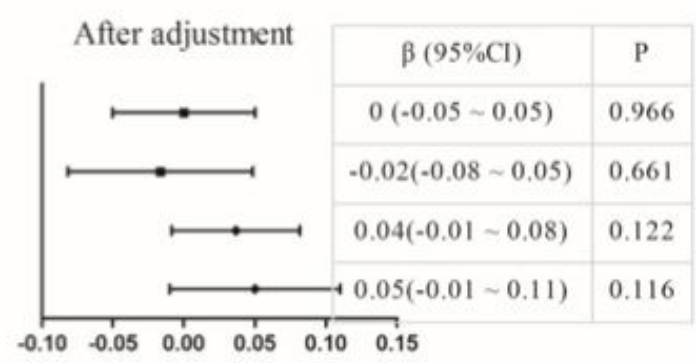

- HADS-D

- HADS-A

\section{Figure 3}

The effects of BCVA of the better-/worse- seeing eyes (A) and MD of the less-/more- damaged eyes (B) as an independent variable on HADS-A and HADS-D by multivariate linear regression analysis without (Crude) or with adjustment (After adjustment) for socio-demographic, clinical variables and the composite score of NEI VFQ-25. BCVA: best corrected visual acuity; MD: mean deviation; HADS-A: Hospital Anxiety and Depression Scale-Anxiety; HADS-D: Hospital Anxiety and Depression ScaleDepression; NEI VFQ-25: National Eye Institute Visual Function Questionnaire 25.

\section{Supplementary Files}

This is a list of supplementary files associated with this preprint. Click to download.

- rawdata.xlsx 